\title{
Evidence of Osteogenic Regulation in Calcific Porcine Aortic Valves
}

\author{
Xiaohong Liu, MD, PhD, Hao Wu, BSc, Yifan Bai, MD, PhD, Dejun Gong, BSc, Cuiping Xia, BSc, \\ Qin Li, BSc, Fanglin Lu, MD, PhD, Zhiyun Xu, MD, PhD \\ Department of Cardiothoracic Surgery, Changhai Hospital, Second Military Medical University, Shanghai, China
}

\section{ABSTRACT}

Background: Chemically cross-linked animal tissues, such as porcine aortic valves (PAVs) have many documented advantages over mechanical valves. However, calcification is the major underlying pathologic process that results in bioprosthetic valve failure. Recently, several reports described the expression of noncollagenous bone matrix proteins in bioprosthetic valves and suggested an actively regulated process of tissue repair.

Methods: Thirty-one explanted PAVs with evidence of calcification were collected and examined for the protein expression implicated in myofibroblast activation, osteoblast differentiation, and bone matrix deposition by using immunohistochemistry.

Results: The mean duration that PAVs were implanted was $11.5 \pm 5.6$ years, ranging from 12 months to 28 years. Pearson correlation analysis showed a significant relationship between the duration and valvular calcification $(r=0.3818$, $P=.034)$. The number of vimentin-positive mesenchymal cells in explanted PAVs was significantly lower than that of unused PAVs $(P<.01)$. However, increased expression of $\alpha$-smooth muscle actin $(\alpha-\mathrm{SMA})(P<.01)$, proliferating cell nuclear antigen (PCNA, $P<.01)$, Cbfa1/Runx2 $(P<.01)$, osterix $(P=.0126)$, bone sialoprotein (BSP, $P<.01)$, osteocal$\operatorname{cin}(P<.01)$, and osteopontin $(P<.01)$ was found in explanted PAVs. Immunohistochemical staining of alkaline phosphatase (ALP) and osteocalcin was negative in the unused PAVs. In explanted PAVs, the expression level of these 2 proteins was also significantly increased.

Conclusions: Our results support the view that PAV calcification is an actively regulated process with osteogenic signaling activation.

\section{INTRODUCTION}

Surgical replacement of diseased heart valves using mechanical or biological valves is now commonplace and

Received May, 2, 2018; accepted fuly, 3, 2018.

Co-first authors: Xiaohong Liu, Hao Wu, and Yifan Bai

Correspondence: Fanglin Lu, Department of Cardiothoracic Surgery, Changhai Hospital, Second Military Medical University, 168, Changhai Rd. Shanghai, 200433, China; 0086-021-81873439; fax: 0086-021-65490979 (e-mail: FanglL_xxwk@126.com). enhances survival and quality of life for many patients. Mechanical valves have a functional life span of at least 25 years but require lifelong anticoagulation therapy to minimize risks of thrombosis and embolism [Hammermeister 2000; Khan 2001; Minakata 2015]. Bioprosthetic valves, especially homografts, have better hemodynamic characteristics and avoid long-term anticoagulation therapies, but their supply is limited [Birkmeyer 2000; Saleeb 2014]. As an alternative, chemically cross-linked animal tissues, such as porcine aortic valves (PAVs), are used widely and have been confirmed to have superior hemodynamic and thromboresistant properties compared to mechanical valves [Skowasch 2006; Alsoufi 2009]. However, these bioprosthetic valves fabricated from animal tissues have a higher rate of failure. Within 10 years following implantation, approximately one third of bioprosthetic valves require replacement, increasing to two thirds by 15 years [Schoen 2005].

Calcification is the major underlying pathologic process that results in bioprosthetic failure. It is generally considered to be related to host factors (eg, young age, uremia, hyperparathyroidism), tissue fixation conditions (eg, chemical cross-linking), immune response, and mechanical stress [Levy 1983; Nimni 1988]. However, these mechanisms of calcification, although concordant with the known data on cellular location of calcium phosphate deposition, do not fully explain the concurrent process of bioprosthetic calcification. Recently, emerging evidence has demonstrated the

Table 1. Antibodies Used in This Study*

\begin{tabular}{lcccc}
\hline Antibodies & Source & Catalogue & Dilution & Retrieval \\
\hline Vimentin & Santa Cruz & Sc6260 & $1: 700$ & Microwave \\
Q-SMA & Affinity & Af1032 & $1: 200$ & Microwave \\
PCNA & abcam & ab29 & $1: 20000$ & Water bath \\
Cbfa1/Runx2 & abcam & ab76956 & $1: 500$ & Water bath \\
Osterix & abcam & ab22552 & $1: 500$ & Water bath \\
ALP & abcam & ab95462 & $1: 500$ & Water bath \\
Osteopontin & abcam & ab8448 & $1: 200$ & Water bath \\
Osteocalcin & abcam & ab13420 & $10 \mu \mathrm{g} / \mathrm{mL}$ & Water bath \\
BSP & abcam & ab52128 & $1: 100$ & Water bath \\
\hline
\end{tabular}

* $\alpha$-SMA, $\alpha$-smooth muscle actin; PCNA, proliferating cell nuclear antigen; ALP, alkaline phosphatase; BSP, bone sialoprotein. 

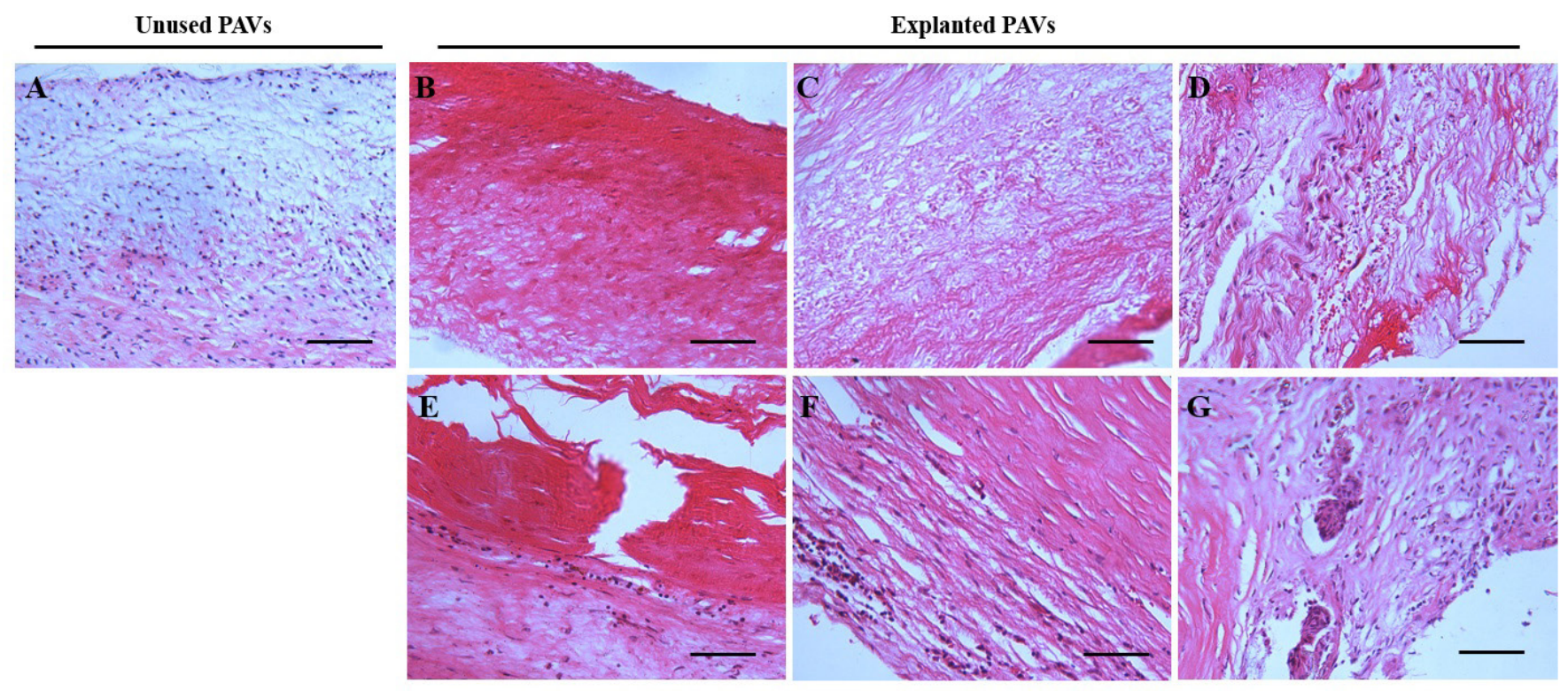

Figure 1. Histological features of unused and explanted PAVs. Unused PAVs demonstrated layered histology (A). Devitalized cells (B), degenerative and fragmented changes of collagen fibers (C), infiltration of living host mesenchymal cell (D), infiltration of chronic inflammatory cells (E), fibrous pannus (F), and foreign-body giant cell response $(\mathrm{G})$ could be found in explanted PAVs. Bar $=100 \mu \mathrm{m}$.
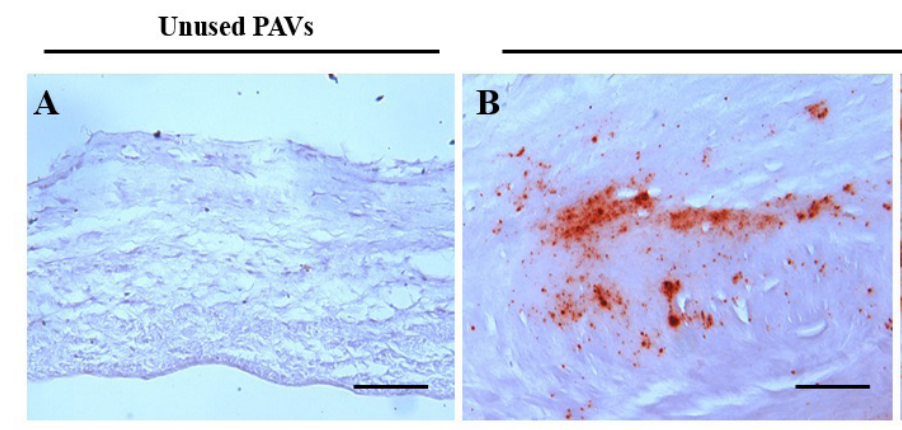

\section{Explanted PAVs}

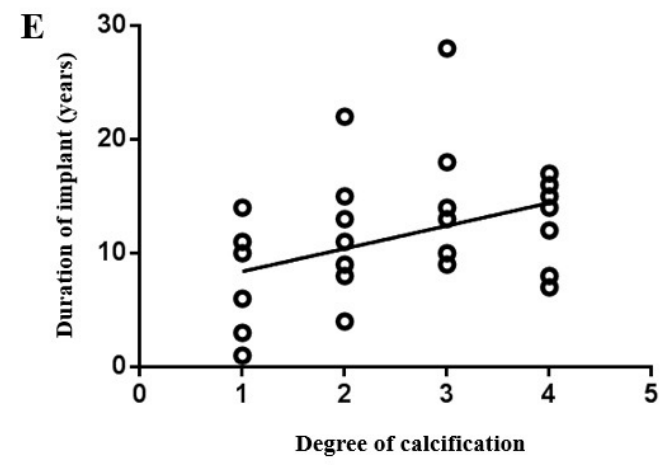

Figure 2. Alizarin red staining of unused and explanted PAVs. Unused PAVs were negative for alizarin red staining (A). Focal (B), mild (C), and a large amount of (D) calcium deposition could be found in explanted PAVs. Correlation of the duration and valvular calcification was confirmed by a Pearson correlation analysis $(E)$. Bar $=100 \mu \mathrm{m}$.

expression of noncollagenous matrix proteins in calcific bioprosthetic valves [Srivatsa 1997; Shetty 2006; Butany 2007], suggesting that bioprosthetic calcification is also an active, cell-mediated process.
In this study, we hypothesized that the implantation of glutaraldehyde cross-linked PAVs attract and activate host nonbone mesenchymal cells and induce them into osteoblast-like cells. These "new" cells would express and secret 

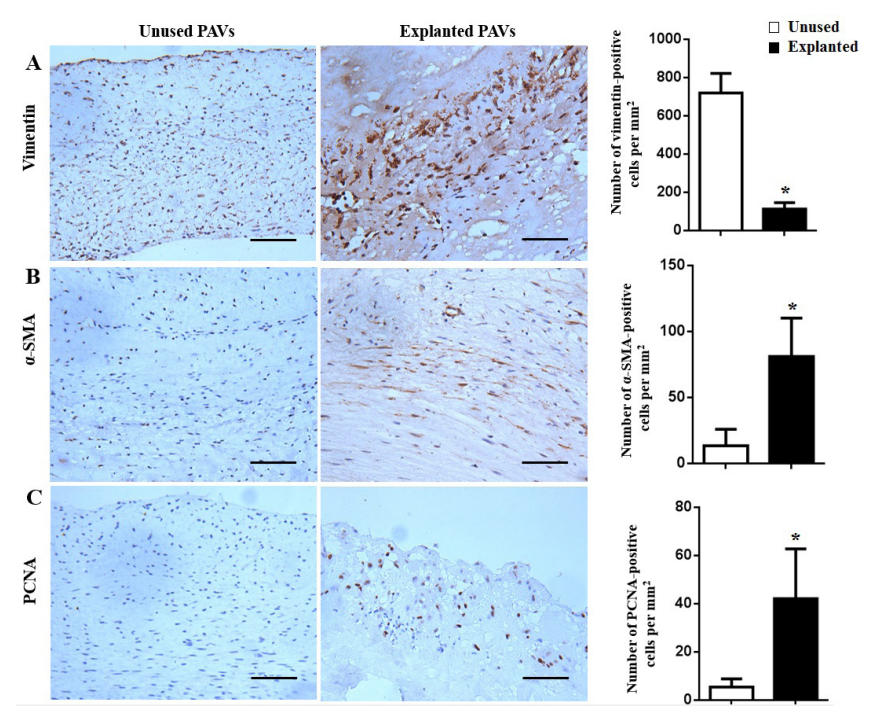

Figure 3. Myofibroblast activation in explanted PAVs. Immunohistochemical staining was performed to detect vimentin $(A), \alpha-S M A(B)$, and PCNA-positive (C) cells. Bar $=100 \mu \mathrm{m} .{ }^{*} P<.05$.

bone-associated proteins under the control of specific transcription factors. To evaluate this hypothesis, we collected 31 explanted PAVs and examined the protein expression implicated in myofibroblast activation, osteoblast differentiation, and bone matrix deposition.

\section{MATERIALS AND METHODS}

\section{Patients and Samples}

This investigation was a retrospective chart review study. The use of tissues explanted from humans was approved by the institutional committee for human research of Changhai Hospital, and informed consent was obtained from all the subjects. Explanted PAV cases were identified from the pathology archives of the Department of Cardiothoracic Surgery, Changhai Hospital, between 1964 and 2010. Cases that did not show evidence of calcific degeneration, had active endocarditis, or had unknown implantation-resection interval were excluded. The remaining cases were then individually assessed for patient demographic data, reason for valve replacement, implant duration, and pathologic data. In addition, 10 unused PAV specimens were collected and served as controls.

\section{Histological Analysis}

Decalcified and fixed tissues were processed for routine paraffin embedding. PAVs were excised vertically to the base at the midpoint. Paraffin-embedded materials were cut into 5 - $\mu \mathrm{m}$ sections and deparaffinized for hematoxylin and eosin (H\&E) staining. Alizarin red staining was used to assess calcification in PAVs. The degree of calcification was graded by inspection of gross and histological analysis: grade 1 had small, isolated calcific nodules; grade 2, moderate, patchy nodules up to $1 \mathrm{~mm}$ in length; grade 3 , severe nodules up to 2 $\mathrm{mm}$ in length; grade 4, very severe, diffuse nodules capable of puncturing through the cusp surface.

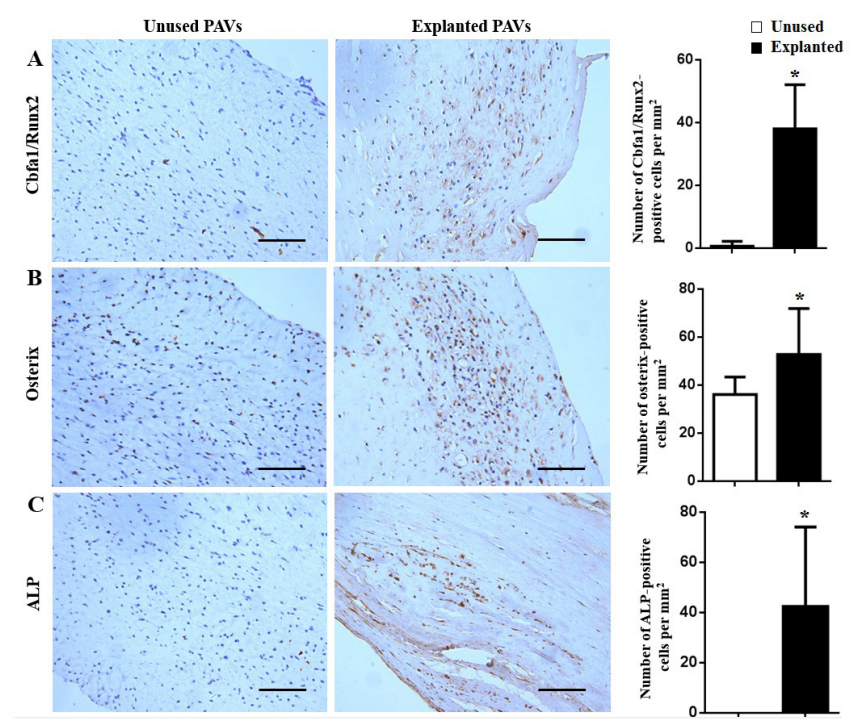

Figure 4. Osteoblastic differentiation in explanted PAVs. Immunohistochemical staining was performed to detect Cbfa1/Run $\times 2(A)$, osterix (B), and ALP-positive (C) osteoblasts. Bar $=100 \mu \mathrm{m} .{ }^{*} P<.05$.

\section{Immunobistochemical Analysis}

Paraffin-embedded materials were cut into 5 - $\mu \mathrm{m}$ sections and deparaffinized. Endogenous peroxidase was blocked with $0.3 \%$ hydrogen peroxide for 30 minutes. Antigen retrieval was accomplished by either of a 15-minute microwave pretreatment or a 30-minute water bath pretreatment in citrate buffer (0.01 M, pH 6.0). After incubation with protein block serum-free (Fuzhou Maixin Biotechnology Development Co., Fuzhou, China), sections were incubated with primary antibodies (Table 1) diluted with antibody diluents (Fuzhou Maixin) overnight at $4^{\circ} \mathrm{C}$. Subsequently, they were incubated with the biotinylated secondary antibody and streptavidinperoxidase complex at room temperature for 15 minutes each (streptavidin-peroxidase kit, Fuzhou Maixin) and visualized with 3,3'-diaminobenzidine (DAB, Fuzhou Maixin ). Nuclear counterstaining was with hematoxylin.

\section{Semiquantitative Evaluation of Immunobistochemical Labeling}

The immunohistochemical findings were evaluated by 2 pathologists, who were blinded to all data. For evaluating vimentin, $\alpha$-smooth muscle actin ( $\alpha$-SMA), proliferating cell nuclear antigen (PCNA), alkaline phosphatase (ALP), Cbfa1/ Runx2, and osterix, the numbers of immunoreactive cells for each of the antigens (per $\mathrm{mm}^{2}$ ) were counted. Immunoreactivity score for osteopontin, osteocalcin, and bone sialoprotein (BSP) was calculated by multiplying the scores for intensity (1-4) and extensity (0\%-100\%) as previously published. Sections with no staining and weak, moderate, and intense staining were assigned scores of $0,1,2$, and 3, respectively [Detre 1995].

\section{Statistical Analysis}

Data are expressed as the mean \pm standard deviation, and statistical analysis was performed with GraphPad software 

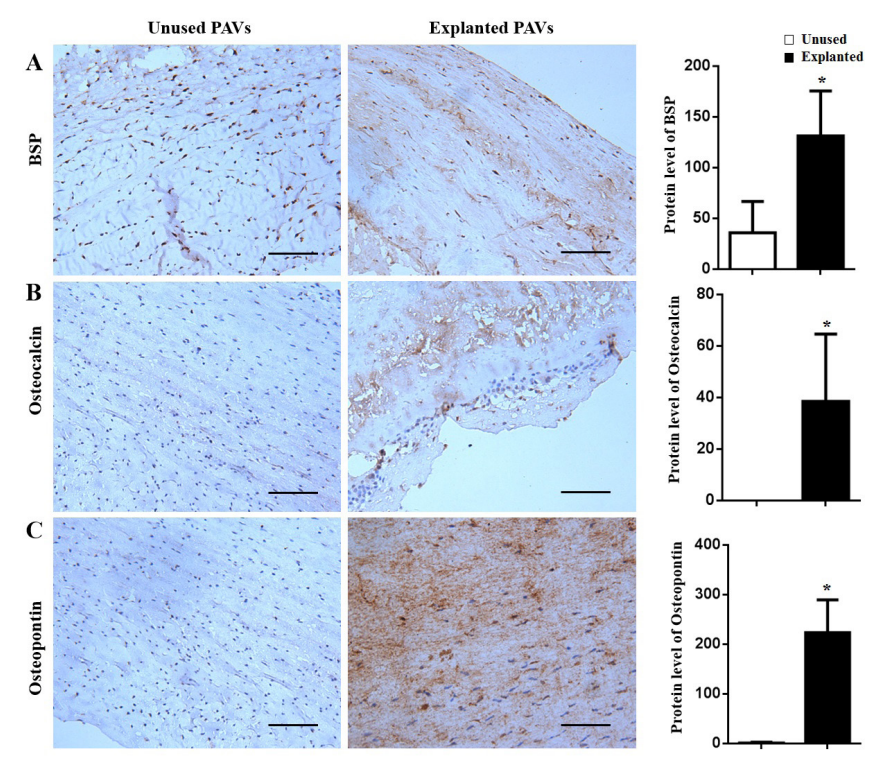

Figure 5. Noncollagenous bone matrix deposition in explanted PAVs. Immunohistochemical staining was performed to detect BSP (A), osteopontin (B), and osteocalcin-positive (C) matrix. Bar $=100 \mu \mathrm{m} .{ }^{*} P<.05$.

(GraphPad Software, La Jolla, CA, USA). Normally distributed variables were analyzed by using the Student $t$ test, and nonnormally distributed variables were analyzed by using the Wilcoxon signed rank test. Pearson correlation analysis was used to examine the relationships between continuous variables. $P$ value $<.05$ was considered statistically significant for all tests.

\section{RESULTS}

\section{Patients}

A total of 31 explanted PAVs were obtained from patients who underwent reoperation due to calcific degeneration of PAVs at Changhai Hospital from 1964 to 2010. As shown in Table 2, 8 patients were male and 23 were female. The mean age of patients at the time of valve implantation ranged from 21 to 67 years $(37.8 \pm 12.8$ years $)$, whereas the mean age at explantation ranged from 31 to 76 years $(49.3 \pm 11.0$ years). The mean duration of implant was $11.5 \pm 5.6$ years, ranging from 12 months to 28 years. Four PAVs (13\%) were explanted $\leq 5$ years after implantation; $10(32 \%)$ were explanted between 6 and 10 years after implantation; $12(39 \%)$ were explanted between 11 and 15 years after implantation; 3 (10\%) were explanted between 16 and 20 years after implantation; and 2 $(6 \%)$ were explanted $>20$ years after implantation.

\section{Histopathological Features}

Unused PAVs consisted of 3 layers: fibrosa, spongiosa, and ventricularis. Valvular interstitial cells were the most abundant cells in the unused PAVs and were dispersed throughout all 3 layers (Figure 1A). All explanted PAVs demonstrated dense and nonlayered histology, with extensive native cell loss (Figure 1B). Collagen fibers were degenerative and fragmented (Figure 1C). Host mesenchymal cells infiltrated and penetrated deeper layer of PAVs (Figure 1D). Scattered

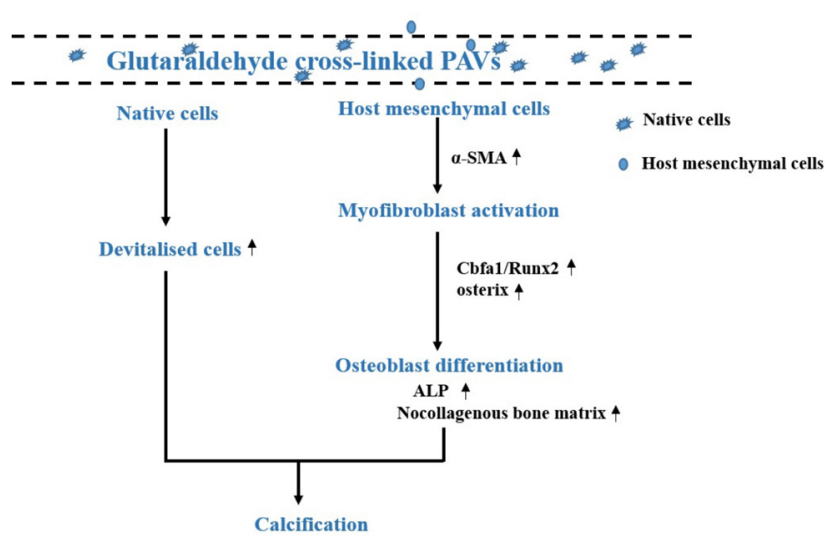

Figure 6. Schematic depiction of cell-mediated calcification in PAVs. On the one hand, devitalized cells within PAVs locally concentrated calcium and phosphate. On the other hand, glutaraldehyde cross-linked PAVs attracted host mesenchymal cells and activated them into myofibroblasts. Subsequently, $\alpha$-SMA-positive myofibroblasts were induced into osteoblast-like cells. These "new" cells expressed ALP and secreted boneassociated proteins (such as osteopontin, osteocalcin and BSP) under the control of specific transcription factors (such as Cbfa1/Runx2 and osterix), leading to the deposition of calcium and phosphate.

mononuclear infiltrates (Figure 1E) were observed in 2 cases though neither one of these cases was associated with infectious microorganisms. Mild or moderate fibrous pannus (Figure 1F) was found in all explanted PAVs, and chronic inflammatory cell infiltrates could be seen. One case showed a foreign-body giant cell response to the PAV tissues (Figure $1 G)$. The presence of osseous metaplasia and chondromatous metaplasia was not observed in any of the explanted PAVs.

\section{Calcification of Explanted PAVs}

Seven of the explanted PAVs (23\%) showed evidence of calcification on gross inspection. On histological examination, alizarin red staining demonstrated calcification in explanted PAVs ranging from focal to a large amount of calcium deposition (Figures 2B-D), whereas unused PAVs were negative for alizarin red staining (Figure 2A). Pearson correlation analysis (Figure 2E) showed a significant relationship between the duration that PAVs were implanted and valvular calcification $(\mathrm{r}=0.3818, P=.034)$.

\section{Evidence of Myofibroblast Activation in Explanted PAVs}

Cellular density was significantly decreased in explanted PAVs compared to unused PAVs. The number of vimentinpositive mesenchymal cells in explanted PAVs was significantly lower than that of unused PAVs (Figure 3A, 113.0 \pm 33.2 versus $718.7 \pm 103.0 ; P<.01)$. In contrast, $\alpha$-SMApositive myofibroblast activation was very low in unused PAVs, but robustly increased in explanted PAVs (Figure 3B, $13.4 \pm 12.5$ versus $80.9 \pm 29.2 ; P<.01)$. Furthermore, in 6 explanted PAVs, $\alpha$-SMA-positive myofibroblasts formed clusters close to the calcific nodules. Immunohistochemistry evaluation of PCNA showed a significant increase in proliferation activity of cells in explanted PAVs as compared with unused PAVs (Figure 3C, $5.4 \pm 3.4$ versus $42.1 \pm 20.7 ; P<.01$ ). 
Table 2. Demographics and Patient Data for Explanted PAV Cases

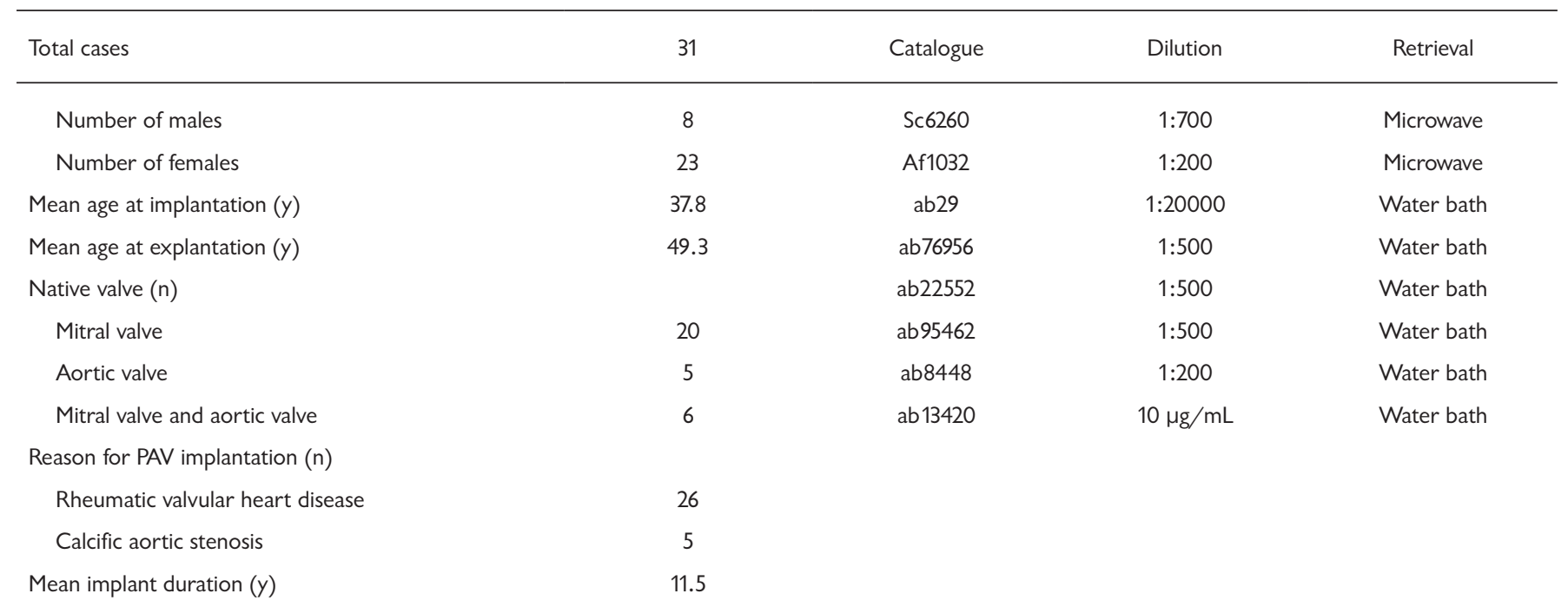

\section{Evidence of Osteoblastic Differentiation in Explanted PAVs}

Cbfa1/Runx2 and osterix are 2 osteoblast specific transcription factors. As shown in Figure 4, the protein expression of these 2 transcription factors was weak in unused PAVs, whereas increased expression of Cbfa1/Runx2 (Figure 4A) and osterix (Figure 4B) was found in explanted PAVs. The number of Cbfa1/Runx2-positive cells in explanted PAVs was significantly higher than that in unused PAVs $(0.6 \pm 1.6$ versus $38.0 \pm 14.1 ; P<.01)$. Similarly, osterix-positive cells in explanted PAVs was significantly higher than that in unused PAVs $(36.1 \pm 7.2$ versus $52.7 \pm 19.1 ; P=.0126)$. ALP is an earlier marker for osteoblast differentiation and a Ca2+-binding glycoprotein. Immunohistochemistry showed that ALP was positive in all explanted PAV cases (Figure 4C), whereas it was negative in all unused PAVs.

\section{Evidence of Noncollagenous Bone Matrix Deposition in Explanted PAVs}

BSP was diffusely expressed and present throughout the fibrosa, spongiosa, and ventricularis, including in valvular endothelial cells, valvular interstitial cells, and extracellular matrix fibers (Figure 5A). Immunohistochemical staining of osteocalcin was negative in the unused PAVs (Figure 5B). Osteopontin was weakly expressed in the unused PAVs and mainly confined to valvular interstitial cells (Figure 5C). In explanted PAVs, the expression level of osteopontin, osteocalcin, and BSP was significantly increased (Figure 5). They were present throughout the valves, including cells and extracellular matrix fibers. Quantitatively, significant differences in osteopontin $(1.1 \pm 1.9$ versus $223.2 \pm 66.1 ; P<.01)$ and BSP $(35.9 \pm 30.8$ versus $131.1 \pm 44.4 ; P<.01)$ were found between unused and explanted PAVs.

\section{DISCUSSION}

Ectopic calcification, once believed to be a passive degenerative process, is now viewed as an active process, with evidence suggesting that it follows a mechanism similar to that of bone formation [Brandenburg 2017; Ciavarella 2017; Pillai 2017]. In this study, our observations using a panel of antibodies against several ossification regulatory proteins support the assumption that bioprosthetic calcification, rather than being attributable to time-dependent passive calcium deposition, is a tightly regulated and cell-mediated active process (Figure 6).

Recently, a growing number of studies of have identified the abnormal expression of noncollagenous matrix proteins in bioprosthetic valves [Shen 1997; Srivatsa 1997; Shetty 2006]. These matrix proteins such as osteocalcin, osteonectin, and osteopontin may regulate calcification by actively coordinating the recruitment of cellular elements and calcium phosphate required for calcification. Comparable results were obtained in our studies in which we found several noncollagenous matrix proteins, including calcification activators and inhibitors, were accumulated in explanted PAVs. Osteopontin is a secreted, integrin-binding glycophosphoprotein that binds calcium and mediates cell attachment [Singh 2014]. It is present in calcific vessels [Lomashvili 2004; Cho 2009] and valves [Yu 2009] and demonstrates itself as a calcification inhibitor. It has been confirmed that osteopontin inhibits mineralization of vascular smooth muscle cells by binding to the mineralized crystal surface [Jono 2000]. Osteocalcin is the most abundant noncollagenous protein present in bone and accumulates in sites of ectopic calcification [Lian 1988]. It was generally thought that osteocalcin inhibits crystal growth and limits bone formation [Levy 1983]. BSP is a secreted glycoprotein and is known to promote nucleation of hydroxyapatite crystals in vitro, and the protein binds to hydroxyapatite via long acidic amino acid stretches [Zhou 1995]. Reduced production of BSP is consistent with reduced matrix calcification in NCad $\Delta$ C-expressing cultures [Cheng 2000].

Studies in different species have shown that various osteoblasts or osteoblast-like cells serve as a cellular source of noncollagenous matrix proteins. In general, bioprosthetic valves 
are treated with glutaraldehyde, which increases tissue durability and devitalizes valvular native cells [Schoen 2005]. Accordingly, it has been suggested that osteoblast-like cells in bioprosthetic calcification originated from host mesenchymal cells. Although the origin of these osteoblast-like cells within the explanted PAV was not clearly defined in our study, we speculated that activated myofibroblasts might undergo cell differentiation and express an osteoblast-like phenotype. In support of this speculation, we found that explanted PAVs were composed of $\alpha$-SMA-positive myofibroblasts, which in some areas formed cellular clusters close to calcific regions. Myofibroblasts are active synthetic cells that take part in active tissue remodeling; therefore, the presence of $\alpha$-SMA -positive cells in explanted PAVs may reflect the active synthesis of extracellular matrix in areas in which calcific matrix is deposited.

The differentiation of osteoblasts from mesenchymal precursors requires a series of cell fate decisions controlled by a hierarchy of transcription factors. Cbfa1/Runx2, a runt family transcription factor, is required for early mesenchymal commitment to osteoblastic lineages. Cbfa1/Runx2-/- mice show complete lack of both intramembranous and endochondral ossification because of the absence of osteoblast differentiation [Komori 2005]. Elevated Cbfa1/Runx2 expression has been found in calcific vascular and valvular diseases [Shao 2006; Miller 2010]. Osterix, a zinc-finger-containing transcription factor that is known to be downstream of Cbfa1/ Runx2 [Nishio 2006], is expressed in osteoblasts of all endochondral and membranous bones. Osterix-/- mice also show the complete lack of both intramembranous and endochondral ossification because of the absence of osteoblast differentiation [Nakashima 2002]. ALP, a key marker for osteoblast activity, is a membrane-associated enzyme that converts organic phosphate into orthophosphate to form hydroxyapatite crystals [van Straalen 1991]. A high level of bone ALP activity is necessary to ensure adequate mineralization of bone, as inhibiting the activity of this enzyme is associated with impaired skeletal development [Calero 1999]. In the present study, we have observed in explanted PAVs a population of cells expressing Runx2, osterix, and ALP, indicating the presence of cells with an osteoblast-like phenotype.

In conclusion, these results confirmed that PAV calcification was not just a purely degenerative and passive process. In some respects, the process was a cell-mediated biologically regulated process that-like osteogenesis-involves both activators and inhibitors. It is no doubt the imbalance between these calcification activators and inhibitors may result in the formation of ectopic calcification at a given site.

\section{ACKNOWLEDGMENTS}

The authors have no conflicts of interest. The present study was supported by a grant from the National Key Research and Development Program of China (no. 2016YFC1100900).

\section{REFERENCES}

Alsoufi B, Manlhiot C, McCrindle BW, et al. 2009. Aortic and mitral valve replacement in children: is there any role for biologic and bioprosthetic substitutes? Eur J Cardiothorac Surg 36:84-90; discussion 90.

Birkmeyer NJ, Birkmeyer JD, Tosteson AN, Grunkemeier GL, Marrin CA, O'Connor GT. 2000. Prosthetic valve type for patients undergoing aortic valve replacement: a decision analysis. Ann Thorac Surg 70:1946-52.

Brandenburg VM, Reinartz S, Kaesler N, et al. 2017. Slower progress of aortic valve calcification with vitamin $\mathrm{K}$ supplementation: results from a prospective interventional proof-of-concept study. Circulation 135:2081-3.

Butany J, Nair V, Leong SW, Soor GS, Feindel C. 2007. CarpentierEdwards Perimount valves--morphological findings in surgical explants. J Card Surg 22:7-12.

Calero JA, Muñoz MT, Argente J, et al. 1999. A variation in Bone Alkaline Phosphatase levels that correlates positively with bone loss and normal levels of aminoterminal propeptide of collagen I in girls with anorexia nervosa. Clin Chim Acta 285:121-9.

Cheng SL, Shin CS, Towler DA, Civitelli R. 2000. A dominant negative cadherin inhibits osteoblast differentiation. J Bone Miner Res $15: 2362-70$

Cho HJ, Cho HJ, Kim HS. 2009. Osteopontin: a multifunctional protein at the crossroads of inflammation, atherosclerosis, and vascular calcification. Curr Atheroscler Rep 11:206-13.

Ciavarella C, Gallitto E, Ricci F, Buzzi M, Stella A, Pasquinelli G. 2017. The crosstalk between vascular MSCs and inflammatory mediators determines the pro-calcific remodelling of human atherosclerotic aneurysm. Stem Cell Res Ther 8:99.

Detre S, Saclani Jotti G, Dowsett M. 1995. A “quickscore" method for immunohistochemical semiquantitation: validation for oestrogen receptor in breast carcinomas. J Clin Pathol 48:876-8.

Hammermeister K, Sethi GK, Henderson WG, Grover FL, Oprian C, Rahimtoola SH. 2000. Outcomes 15 years after valve replacement with a mechanical versus a bioprosthetic valve: final report of the Veterans Affairs randomized trial. J Am Coll Cardiol 36:1152-8.

Jono S, Peinado C, Giachelli CM. 2000. Phosphorylation of osteopontin is required for inhibition of vascular smooth muscle cell calcification. J Biol Chem 275:20197-203.

Khan SS, Trento A, DeRobertis M, et al. 2001. Twenty-year comparison of tissue and mechanical valve replacement. J Thorac Cardiovasc Surg 122:257-69.

Komori T. 2005. Regulation of skeletal development by the Runx family of transcription factors. J Cell Biochem 95:445-53.

Levy RJ. 1983. Biologic determinants of dystrophic calcification and osteocalcin deposition in glutaraldehyde-preserved porcine aortic valve leaflets implanted subcutaneously in rats. Am J Pathol 113:143-55.

Levy RJ, Gundberg C, Scheinman R. 1983. The identification of the vitamin $\mathrm{K}$-dependent bone protein osteocalcin as one of the gamma-carboxyglutamic acid containing proteins present in calcified atherosclerotic plaque and mineralized heart valves. Atherosclerosis 46:49-56.

Lian JB, Gundberg CM. 1988. Osteocalcin. Biochemical considerations and clinical applications. Clin Orthop Relat Res 226:267-91.

Lomashvili KA, Cobbs S, Hennigar RA, Hardcastle KI, O’Neill WC. 2004. Phosphate-induced vascular calcification: role of pyrophosphate and osteopontin. J Am Soc Nephrol 15:1392-401.

Miller JD, Weiss RM, Serrano KM, et al. 2010. Evidence for active regulation of pro-osteogenic signaling in advanced aortic valve disease. 
Arterioscler Thromb Vasc Biol 30:2482-6.

Minakata K, Tanaka S, Okawa Y, et al. 2015. Twenty-year outcome of aortic valve replacement with St. Jude Medical mechanical valves in Japanese patients. Circ J 79:2380-8.

Nakashima K, Zhou X, Kunkel G, et al. 2002. The novel zinc fingercontaining transcription factor osterix is required for osteoblast differentiation and bone formation. Cell 108:17-29.

Nimni ME, Bernick S, Cheung DT, et al. 1988. Biochemical differences between dystrophic calcification of cross-linked collagen implants and mineralization during bone induction. Calcif Tissue Int 42:313-20.

Nishio Y, Dong Y, Paris M, O'Keefe RJ, Schwarz EM, Drissi H. 2006. Runx2-mediated regulation of the zinc finger Osterix/Sp7 gene. Gene 372:62-70.

Pillai ICL, Li S, Romay M, et al. 2017. Cardiac fibroblasts adopt osteogenic fates and can be targeted to attenuate pathological heart calcification. Cell Stem Cell 20:218-232.e5.

Saleeb SF, Newburger JW, Geva T, et al. 2014. Accelerated degeneration of a bovine pericardial bioprosthetic aortic valve in children and young adults. Circulation 130:51-60.

Schoen FJ, Levy RJ. 2005. Calcification of tissue heart valve substitutes: progress toward understanding and prevention. Ann Thorac Surg 79:1072-80.

Shao JS, Cai J, Towler DA. 2006. Molecular mechanisms of vascular calcification: lessons learned from the aorta. Arterioscler Thromb Vasc Biol 26:1423-30.
Shen M, Marie P, Farge D, et al. 1997. Osteopontin is associated with bioprosthetic heart valve calcification in humans. C R Acad Sci III $320: 49-57$

Shetty R, Pepin A, Charest A, et al. 2006. Expression of bone-regulatory proteins in human valve allografts. Heart 92:1303-8.

Singh M, Dalal S, Singh K. 2014. Osteopontin: at the cross-roads of myocyte survival and myocardial function. Life Sci 118:1-6.

Skowasch D, Steinmetz M, Nickenig G, Bauriedel G. 2006. Is the degeneration of aortic valve bioprostheses similar to that of native aortic valves? Insights into valvular pathology. Expert Rev Med Devices 3:453-62.

Srivatsa SS, Harrity PJ, Maercklein PB, et al. 1997. Increased cellular expression of matrix proteins that regulate mineralization is associated with calcification of native human and porcine xenograft bioprosthetic heart valves. J Clin Invest 99:996-1009.

van Straalen JP, Sanders E, Prummel MF, Sanders GT. 1991. Bonealkaline phosphatase as indicator of bone formation. Clin Chim Acta 201:27-33.

Yu PJ, Skolnick A, Ferrari G, et al. 2009. Correlation between plasma osteopontin levels and aortic valve calcification: potential insights into the pathogenesis of aortic valve calcification and stenosis. J Thorac Cardiovasc Surg 138:196-9.

Zhou HY, Takita H, Fujisawa R, Mizuno M, Kuboki Y. 1995. Stimulation by bone sialoprotein of calcification in osteoblast-like MC3T3-E1 cells. Calcif Tissue Int 56:403-7. 